\title{
Kinetic energy density functionals for non-periodic systems
}

\section{Citation}

Choly, Nicholas, and Efthimios Kaxiras. 2002. "Kinetic Energy Density Functionals for NonPeriodic Systems." Solid State Communications 121 (5): 281-86. https://doi.org/10.1016/ s0038-1098(01)00500-2.

\section{Permanent link}

http://nrs.harvard.edu/urn-3:HUL.InstRepos:41384126

\section{Terms of Use}

This article was downloaded from Harvard University's DASH repository, and is made available under the terms and conditions applicable to Other Posted Material, as set forth at http:// nrs.harvard.edu/urn-3:HUL.InstRepos:dash.current.terms-of-use\#LAA

\section{Share Your Story}

The Harvard community has made this article openly available.

Please share how this access benefits you. Submit a story.

Accessibility 


\title{
Kinetic energy density functionals for non-periodic systems
}

\author{
Nicholas Choly* and Efthimios Kaxiras \\ Department of Physics and Division of Engineering and Applied Sciences, \\ Harvard University, \\ Cambridge, MA 02138
}

(Dated: October 30, 2018)

\begin{abstract}
Kinetic energy functionals of the electronic density are used to model large systems in the context of density functional theory, without the need to obtain electronic wavefunctions. We discuss the problems associated with the application of widely used kinetic energy functionals to non-periodic systems. We develop a method that circumvents this difficulty and allows the kinetic energy to be evaluated entirely in real space. We demonstrate that the method is efficient $[O(N)]$ and accurate by comparing the results of our real-space formulation to calculations performed in reciprocal space, and to calculations using traditional approaches based on electronic states.

PACS numbers: 71.15.Mb, 71.15.Dx
\end{abstract}

\footnotetext{
* Electronic Address: choly@fas.harvard.edu
} 
Electronic structure calculations based on density functional theory [1, 2] (DFT) and employing an approximate kinetic energy functional[3, 4, 5, 6, 7, 8, 9] have been shown to yield accurate energetics for a variety of physical systems, with a considerably smaller expenditure of computer effort than traditional schemes. A drawback of many existing kinetic energy functionals is the necessity to evaluate them in part in reciprocal space. The benefits of doing electronic structure calculations exclusively in real space are several. Foremost among them is their ability to simulate efficiently isolated systems, whereas reciprocal space methods would require a large supercell with a significant portion of the volume devoted to uninteresting vacuum. A direct extension of this feature is the possibility of using arbitrary boundary conditions rather than the strict periodic boundary conditions underlying reciprocal space approaches; this should be of paramount importance in dealing with complex structures which cannot be accommodated by simple periodic boundary conditions, such as dislocations, cracks, etc. Finally, real space methods can be readily parallelized for efficient computations on parallel computer architectures.

In this article we examine the reasons for the evaluation in reciprocal space of many kinetic energy functionals and propose a new functional form that can reproduce the energetics of those functionals but does not require any reciprocal space evaluations. The performance of the new functionals is evaluated and compared to the existing reciprocal space functionals as well as to the traditional approaches based on the calculation of electronic states.

DFT in its usual guise consists of solving the Kohn-Sham (KS) equations[2]. The fictitious $\mathrm{KS}$ wavefunctions allow the exact evaluation of $T_{s}[\rho]$, the kinetic energy of non-interacting fermions with a density $\rho(\mathbf{r})$. Recent calculations have shown that for certain systems the solution of the KS equations, usually the most computationally demanding part of electronic structure calculations, can be bypassed using an approximate form for $T_{s}[\rho][8]$. A fair number of approximate non-interacting kinetic energy functionals have been proposed for use within such orbital-free methods. A subset of them are similar in form 4, 5, 6, 7, 8, sharing the following common traits:

1. A major ingredient of $T_{s}[\rho]$ is the Thomas-Fermi (TF) energy [10]:

$$
T_{T F}[\rho]=C_{T F} \int \rho^{5 / 3}(\mathbf{r}) d \mathbf{r}
$$

where $C_{T F}=\frac{3}{10}\left(3 \pi^{2}\right)^{2 / 3}$. Here and throughout this article atomic units $\left(\hbar=m_{e}=\right.$ $e=1)$ are employed. The TF energy approximates the kinetic energy in an element of 
space $d \mathbf{r}$ with that of a homogeneous non-interacting electron gas with a density $\rho(\mathbf{r})$. Hence for homogeneous densities $\rho(\mathbf{r})=\rho_{0}, T_{T F}[\rho(\mathbf{r})]$ is exact.

2. Another important contribution is the von Weizsäcker energy [11], given by:

$$
T_{v W}[\rho]=-\frac{1}{2} \int \sqrt{\rho(\mathbf{r})} \nabla^{2} \sqrt{\rho(\mathbf{r})} d \mathbf{r}
$$

It can be readily shown that the von Weizsäcker energy yields the correct kinetic energy for a non-interacting fermion density that consists of a single orbital, i.e. a one- or two-electron density. Also, for any density, the von Weizsäcker energy yields the energy that a system of non-interacting bosons of density $\rho(\mathbf{r})$ would have. This term is a lower bound of $T_{s}[\rho][13]$.

3. The response of a homogeneous non-interacting Fermi gas to a small perturbation is known exactly [12]. This response can be related to the second functional derivative of $T_{s}[\rho]$ evaluated at uniform density $\rho(\mathbf{r})=\rho_{0}$. The Fourier transform of this functional derivative is given by:

$$
\begin{array}{r}
\hat{\mathcal{F}}\left[\left.\frac{\delta^{2} T_{s}}{\delta \rho(\mathbf{r}) \delta \rho\left(\mathbf{r}^{\prime}\right)}\right|_{\rho_{0}}\right]=-\frac{1}{\chi_{\text {Lind }}(q)} \\
\chi_{\text {Lind }}(q)=-\frac{k_{F}}{\pi^{2}}\left[\frac{1}{2}+\frac{1-q^{2}}{4 q} \ln \left|\frac{1+q}{1-q}\right|\right]
\end{array}
$$

where $\chi_{\text {Lind }}(q)$ is the Lindhard response function, $\hat{\mathcal{F}}[f(\mathbf{r})]=\int f(\mathbf{r}) e^{i \mathbf{k} \cdot \mathbf{r}} d \mathbf{r}$ denotes the action of the Fourier transform on the function $f(\mathbf{r}), \mathbf{q}=\mathbf{k} / 2 k_{F}$, and $q=|\mathbf{q}|$. In order to satisfy Eq.(倩) in addition to items 11 and 2 above, the kinetic energy functionals under consideration here include a term $T_{K}[\rho]$, which will be hereafter referred to as the kernel energy:

$$
T_{K}[\rho]=\int f(\rho(\mathbf{r})) K\left(\left|\mathbf{r}-\mathbf{r}^{\prime}\right|\right) g\left(\rho\left(\mathbf{r}^{\prime}\right)\right) d \mathbf{r} d \mathbf{r}^{\prime}
$$

where for the moment $f(\rho), g(\rho)$ are arbitrary functions that can be chosen to satisfy known limits of the exact $T_{s}[\rho]$.

The total kinetic energy functional is taken to be the sum of these terms:

$$
T_{s}[\rho] \simeq T_{T F}[\rho]+T_{v W}[\rho]+T_{K}[\rho]
$$


By plugging Eq.(6) into Eq.(3), it is seen that $T_{s}[\rho]$ exhibits the correct linear response, provided:

$$
\begin{aligned}
& \hat{\mathcal{F}}\left[\left.\frac{\delta^{2} T_{K}}{\delta \rho(\mathbf{r}) \delta \rho\left(\mathbf{r}^{\prime}\right)}\right|_{\rho_{0}}\right]=-\frac{1}{\chi_{\text {Lind }}(q)} \\
& \quad-\hat{\mathcal{F}}\left[\left.\frac{\delta^{2} T_{T F}}{\delta \rho(\mathbf{r}) \delta \rho\left(\mathbf{r}^{\prime}\right)}\right|_{\rho_{0}}\right]-\hat{\mathcal{F}}\left[\left.\frac{\delta^{2} T_{v W}}{\delta \rho(\mathbf{r}) \delta \rho\left(\mathbf{r}^{\prime}\right)}\right|_{\rho_{0}}\right]
\end{aligned}
$$

The functional derivatives of $T_{T F}$ and $T_{v W}$ are well known, and the second functional derivative of $T_{K}$ can, by design, be easily evaluated, so that Eq. (7) takes the form:

$$
\begin{gathered}
\frac{2 k_{F}}{\pi^{2}} f^{\prime}\left(\rho_{0}\right) g^{\prime}\left(\rho_{0}\right) K(q)=\hat{K}(q) \\
\hat{K}(q) \equiv-\frac{k_{F}}{\pi^{2} \chi_{\text {Lind }}(q)}-1-3 q^{2}
\end{gathered}
$$

For any choice of the functions $f$ and $g, K(q)$ can be readily chosen such that the total kinetic energy functional exhibits the correct linear response. The different kinetic energy functionals considered in this paper differ mostly in the choice of the functions $f$ and $g$.

In order to use kinetic energy functionals in actual electronic structure calculations, an algorithm must be developed for their action on discrete representations of the charge density. It is clear that $T_{T F}[\rho(\mathbf{r})]$ and $T_{v W}[\rho(\mathbf{r})]$ can be computed easily and efficiently on a grid in real space:

$$
\begin{gathered}
T_{T F}\left[\left\{\rho_{i}\right\}\right]=\Omega C_{T F} \sum_{i} \rho_{i}^{5 / 3} \\
T_{v W}\left[\left\{\rho_{i}\right\}\right]=-\frac{\Omega}{2} \sum_{i j} \sqrt{\rho_{i}} \Delta_{i j} \sqrt{\rho_{j}}
\end{gathered}
$$

where $\Omega$ is the volume per grid point, $\left\{\rho_{i}\right\}$ denotes a discrete representation of $\rho(\mathbf{r})$ on a grid in real space, and $\Delta_{i j}$ is a discrete representation of the Laplacian operator. In principle, we could also compute the kernel energy $T_{K}[\rho(\mathbf{r})]$ in real space as follows:

$$
T_{K}\left[\left\{\rho_{i}\right\}\right]=\Omega^{2} \sum_{i j} f\left(\rho_{i}\right) K\left(\left|\mathbf{r}_{j}-\mathbf{r}_{j}\right|\right) g\left(\rho_{j}\right)
$$

However, this is impractical because of the specific form of $K(r) . K(r)$ is the Fourier transform of $K(q)$ (see Eq.(9)), which is a non-trivial Fourier transform. Herring [13] has shown 
how it may be evaluated numerically. The quantity $K(r)$ is shown in Fig. 1, indicating that $K(r)$ does not decay rapidly. In order to evaluate

$$
\int K\left(\left|\mathbf{r}-\mathbf{r}^{\prime}\right|\right) h\left(\mathbf{r}^{\prime}\right) d \mathbf{r}^{\prime}
$$

accurately, the integral needs to be evaluated over a sphere centered at $\mathbf{r}$ of a radius equal to $\lambda$, with $\lambda$ a large value. Such a convolution integral can be computed efficiently in reciprocal space:

$$
\hat{\mathcal{F}}\left[\int K\left(\left|\mathbf{r}-\mathbf{r}^{\prime}\right|\right) h\left(\mathbf{r}^{\prime}\right) d \mathbf{r}^{\prime}\right]=\hat{\mathcal{F}}[K(r)] \hat{\mathcal{F}}[h(\mathbf{r})]
$$

Starting from $K(r)$ and $h(\mathbf{r})$ in real space, three Fast Fourier Transforms (FFTs) are required to evaluate the convolution: two forward transformations, and one reverse transformation. The drawback of computing the kinetic energy functional with FFTs is that this approach maps the problem to a periodic tiling of the system of interest. This periodicity can have consequences on the resulting physics that in many cases are undesirable and can lead to erroneous results.

The problem with evaluating the kinetic energy functionals described above in real space lies in the efficient evaluation of convolution integrals. Convolution integrals with a longranged kernel can sometimes be evaluated efficiently in real space in an indirect way. For instance, the electrostatic potential of a charge distribution $\rho(\mathbf{r})$ is the convolution of $\rho(\mathbf{r})$ with the very long-ranged $1 / r$ :

$$
\Phi(\mathbf{r})=-\int \frac{\rho\left(\mathbf{r}^{\prime}\right)}{\left|\mathbf{r}-\mathbf{r}^{\prime}\right|} d \mathbf{r}^{\prime}
$$

The integral in this expression can be computed efficiently in real space by solving the Poisson equation:

$$
\nabla^{2} \Phi(\mathbf{r})=-4 \pi \rho(\mathbf{r})
$$

In general, as an alternative to evaluating the convolution

$$
V(\mathbf{r})=\int G\left(\mathbf{r}-\mathbf{r}^{\prime}\right) f\left(\mathbf{r}^{\prime}\right) d \mathbf{r}^{\prime}
$$

one may equivalently solve the integral equation:

$$
\begin{array}{r}
\int H\left(\mathbf{r}-\mathbf{r}^{\prime}\right) V\left(\mathbf{r}^{\prime}\right) d \mathbf{r}^{\prime}=f\left(\mathbf{r}^{\prime}\right), \\
H(\mathbf{r})=\hat{\mathcal{F}}^{-1}\left[\frac{1}{\hat{\mathcal{F}}[G(\mathbf{r})]}\right] .
\end{array}
$$


In discretized form, this integral equation is a standard linear problem:

$$
H_{i j} V_{j}=f_{i}
$$

which can be solved by an iterative linear solver [14]; the efficiency of such approaches depends on the efficiency of multiplying an arbitrary vector by a matrix. For the case of the Poisson equation, the matrix $H_{i j}$ becomes a discrete representation of the well-localized Laplacian operator, and hence matrix-vector multiplications are efficient. However, when the integral equation that corresponds to the convolution of Eq.(5) is constructed, the resulting function $H_{K}(r)$ is found to be long-ranged. The long-ranged nature of both $K(r)$ and $H_{K}(r)$ is due to the logarithmic divergence of the slope of $\chi_{\text {Lind }}(q)$ at $q=1$, which causes longranged oscillations to appear in its Fourier transform. Thus, matrix-vector multiplications by $H_{i j}=H_{K}\left(\left|\mathbf{r}_{j}-\mathbf{r}_{j}\right|\right)$, and the solution of the integral Eq.(18), are inefficient.

We are therefore interested in developing a method that circumvents these difficulties. As a first step toward this goal, we note that the kernel appearing in the class of kinetic energy functionals under consideration, $\hat{K}(q)$, can be fit well by the rational function:

$$
\tilde{K}(q)=\frac{N_{2} q^{2}+\cdots+N_{2 m} q^{2 m}}{D_{0}+D_{2} q^{2}+\cdots+D_{2 m} q^{2 m}},
$$

with appropriate choices of the real coefficients $N_{i}$ and $D_{i}$. The odd powers of $q$ are omitted because in the Taylor expansions of $\hat{K}(q)$ about 0 and $\infty$ only even powers of $q$ appear. The quality of the fit is shown in Fig. 2 f for several values of $m$. Next we note that $\tilde{K}(q)$ can be separated into terms of the form:

$$
\tilde{K}(q)=\sum_{j=1}^{m} \frac{P_{j} q^{2}}{q^{2}+Q_{j}},
$$

where the $P_{j}$ and $Q_{j}$ are now complex numbers. With this expression, the convolution of a function $f(\mathbf{r})$ with $\tilde{K}(r)$ in reciprocal space becomes:

$$
V(\mathbf{q}) \equiv \tilde{K}(q) f(\mathbf{q})=V_{1}(\mathbf{q})+\cdots+V_{m}(\mathbf{q}),
$$

where

$$
V_{j}(\mathbf{q})=\frac{P_{j} q^{2}}{q^{2}+Q_{j}} f(\mathbf{q}) .
$$

The $V_{i}(\mathbf{r})$ can be computed efficiently in real space by solving:

$$
\begin{aligned}
\left(q^{2}+Q_{j}\right) V_{j}(\mathbf{q}) & =P_{j} q^{2} f(\mathbf{q}) \\
\Rightarrow\left[-\frac{1}{\left(2 k_{F}\right)^{2}} \nabla^{2}+Q_{j}\right] V_{j}(\mathbf{r}) & =-\frac{P_{j}}{\left(2 k_{F}\right)^{2}} \nabla^{2} f(\mathbf{r})
\end{aligned}
$$


that is, $V_{j}(\mathbf{r})$ is the solution of a complex Helmholtz equation. A shortcut in computing the $V_{j}(\mathbf{r})$ results from the fact that $\tilde{K}(q)$, and thus the sum of the $V_{j}(\mathbf{r})$, is purely real. For every pair of coefficients $\left\{P_{j}, Q_{j}\right\}$, another pair $\left\{P_{k}, Q_{k}\right\}=\left\{P_{j}^{*}, Q_{j}^{*}\right\}$ must also appear in the expansion. It follows that $V_{k}(\mathbf{r})=V_{j}^{*}(\mathbf{r})$, and thus only half of the $V_{j}(\mathbf{r})$ need be computed. A generalization of the kinetic energy functional with the form of Eq.(5) has been developed by Wang et al.[9]. These functionals can also be treated in real space with the present method, as discussed in the Appendix.

The following issue regarding the form of the approximation of Eq.(20) deserves further discussion: an important feature of the Lindhard response function lies in the logarithmic singularity in its slope at $q=1$. As discussed above, this singularity manifests itself mathematically in the long-ranged nature of $K(r)$ and $H_{K}(r)$. Not surprisingly, this singularity also has important physical consequences, such as Friedel oscillations and the Kohn effect 15. The approximate kernel $\tilde{K}(q)$ does not exhibit the singularity. It may seem then that from a physical standpoint, $\tilde{K}(q)$ may not adequately describe the kinetic energy of the electron gas. However, it should be noted that in a discrete representation of the problem, the exact singularity at $q=1$ will not be seen. Furthermore, at non-zero electronic temperatures, however small, the singularity in $\chi_{\text {Lind }}(q)$ disappears. Thus, one could think of the fitting form $\tilde{K}(q)$ as representing $K(q)$ for a small but finite electronic temperature. The use of a fictitious, finite electronic temperature is a trick routinely employed to aid numerical convergence in standard DFT calculations of metals.

We have therefore reduced the problem to solving the complex Helmholtz equation, which by itself provides a special challenge due to the fact that the operator is non-Hermitian. Typical iterative methods for solving linear systems, like the conjugate-gradient algorithm, fail for non-Hermitian matrices. The complex Helmholtz equation is an important problem, arising frequently in the context of electrodynamics. Several iterative methods have been developed for the special class of complex symmetric matrices, into which complex Helmholtz operators fall 16, 17. For the present tests, the biconjugate-gradient algorithm, specialized to complex symmetric matrices [16], has been employed to solve Eq.(24). Each iteration of this method requires an amount of computation that scales linearly with the system size; thus, if the number of iterations required to converge a solution of the Helmholtz equation does not vary significantly with the grid size, the entire method for calculating the total energy scales linearly $[O(N)]$ with the system size $(\mathrm{N})$. 
Solving the discretized version of Eq.(24) in real space suffers from another source of inaccuracy, beyond that introduced by the fitting of $\hat{K}(q)$ by $\tilde{K}(q)$. A discretized version of the Laplacian operator must be employed, which, in reciprocal space, deviates from the exact $q^{2}$ behavior. The two sources of error can be easily separated for the purposes of numerical tests. To measure the error due to the approximation of $\hat{K}(q)$ by $\tilde{K}(q)$ alone, the kernel energy $T_{K}[\rho]$ can be computed with the reciprocal space convolution method, Eq.(14), but using $\tilde{K}(q)$ instead of $\hat{K}(q)$. The error due to the use of the discretized Laplacian, in addition to the fitting error, is present when the full real space evaluation method is used. We found that the error due to a fourth-order discrete Laplacian operator was negligible compared to the error introduced by the fitting of $\hat{K}$, and throughout the numerical tests this fourth-order Laplacian was employed. The kinetic energy evaluated with the present real-space method is denoted by $T_{\tilde{K}}[\rho]$, while the kinetic energy evaluated in reciprocal space with $\hat{K}(q)$ is denoted by $T_{K}[\rho]$. The kinetic energies $T_{K}[\rho]$ and $T_{\tilde{K}}[\rho]$ are evaluated and compared for a realistic set of charge densities $\rho(\mathbf{r})$ in Fig. 3. The $T_{K}[\rho]$ used is due to Wang et al. [9], and has parameters $\{\alpha, \beta\}=\frac{5}{6} \pm \frac{\sqrt{5}}{6}$, and for $T_{\tilde{K}}[\rho]$, successive fitting orders

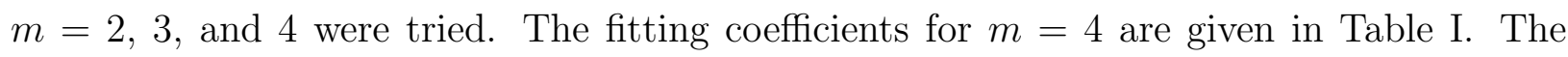
charge densities considered are generated by minimizing the total energy:

$$
\begin{aligned}
E_{t o t}= & T_{T F}[\rho]+T_{v W}[\rho]+T_{K}[\rho]+E_{H}[\rho]+ \\
& E_{\text {ion }}[\rho]+E_{X C}[\rho]
\end{aligned}
$$

where $E_{H}, E_{X C}$, and $E_{\text {ion }}$ are the Hartree, exchange-correlation, and electron-ion interaction terms, for a bulk fcc aluminum system, with a wide range of lattice constants. Aluminum was represented by the Goodwin-Needs-Heine local pseudopotential [18], and exchange and correlation were treated with the LDA[19]. At each lattice constant, after minimizing the electronic energy with the kernel energy represented by $T_{K}[\rho]$, the kernel energy is also computed with $T_{\tilde{K}}[\rho]$ and compared to $T_{K}[\rho]$. As can be seen from Fig. 3, the use of more terms in the fitting (higher $m$ ) results in a smaller deviation, and at $m=4$ the accuracy is quite satisfactory (less than 1\% deviation for the entire range of lattice constants considered.)

We have performed a different set of tests, in which at each value of the lattice constant the total energy with the kernel energy represented by either $T_{K}[\rho]$ or $T_{\tilde{K}}[\rho]$ is minimized with respect to the density. In the test discussed earlier the density was fixed to that obtained from minimization of the total energy using $T_{K}[\rho]$. The present test also differs 
from the last in that the error of the discretized Laplacian is also present in the evaluation of $T_{v W}[\rho]$ in the real space calculations. Thus in this calculation, total energy calculations are performed fully and self-consistently in real space, and are compared with reciprocal space results. The equilibrium lattice constant and the bulk modulus obtained from these calculations are given in Table [1]. As seen from this Table, $m=4$ offers an acceptable level of accuracy: the values obtained with this approximation to the true kernel are essentially the same as those from the exact kernel, differing only by $0.1 \%$ for the lattice constant and by $0.5 \%$ for the bulk modulus.

In conclusion, the convolution integrals that appear in the class of kinetic energy functionals under consideration in this paper cannot be efficiently evaluated directly in real space, and the corresponding inverse integral equations cannot be efficiently solved. The kernels of the convolutions can be approximated by a sum of sub-kernels. This approach makes it possible to evaluate the convolution efficiently in real space by solving the inverse integral equations that correspond to the sub-kernels, which are complex Helmholtz equations. We have demonstrated that this method yields excellent results in numerical tests, in the sense that the error introduced by the real space method is negligible compared to the error inherent in the approximate kinetic energy functionals.

\section{Acknowledgments}

The authors wish to thank Umesh Waghmare and Paul Maragakis for fruitful discussions, and Emily Carter for a critical reading of the manuscript and useful suggestions. Nicholas Choly acknowledges support from an NSF Graduate Research Fellowship. This work was supported in part by a MURI-AFOSR Grant No. F49620-99-1-0272.

\section{APPENDIX A: REAL-SPACE EVALUATION OF KINETIC ENERGY FUNC- TIONALS WITH DENSITY-DEPENDENT KERNELS}

Wang, Govind, and Carter [9] (WGC in the following) have developed a class of kinetic energy functionals that include the Thomas-Fermi and von Weizsäcker terms, as well as a 
term analogous to Eq.(5), but with a density-dependent kernel in the convolution:

$$
\begin{aligned}
& T_{K}[\rho]=C_{T F} \times \\
& \int f(\rho(\mathbf{r})) K\left(\rho(\mathbf{r}), \rho\left(\mathbf{r}^{\prime}\right) ;\left|\mathbf{r}-\mathbf{r}^{\prime}\right|\right) g\left(\rho\left(\mathbf{r}^{\prime}\right)\right) d \mathbf{r} d \mathbf{r}^{\prime}
\end{aligned}
$$

where

$$
K\left(\rho(\mathbf{r}), \rho\left(\mathbf{r}^{\prime}\right) ;\left|\mathbf{r}-\mathbf{r}^{\prime}\right|\right)=K\left(\rho\left(\mathbf{r}^{\prime}\right), \rho(\mathbf{r}) ;\left|\mathbf{r}-\mathbf{r}^{\prime}\right|\right)
$$

Numerical tests indicate that these kinetic energy functionals are more transferable to systems that deviate significantly from the bulk, like surfaces.

Even by utilizing FFTs, a straightforward evaluation of the convolution in Eq. (A1) would require $O\left(N^{2}\right)$ operations, where $N$ is proportional to the size of the system. WGC have demonstrated how this convolution can be efficiently, but approximately, evaluated. By Taylor expanding $K\left(\rho(\mathbf{r}), \rho\left(\mathbf{r}^{\prime}\right) ;\left|\mathbf{r}-\mathbf{r}^{\prime}\right|\right)$ with respect to $\rho(\mathbf{r})$ about some chosen average density $\bar{\rho}$, one obtains:

$$
\begin{aligned}
& K\left(\rho(\mathbf{r}), \rho\left(\mathbf{r}^{\prime}\right) ;\left|\mathbf{r}-\mathbf{r}^{\prime}\right|\right)=K_{0}\left(\left|\mathbf{r}-\mathbf{r}^{\prime}\right|\right) \\
& +K_{1}\left(\left|\mathbf{r}-\mathbf{r}^{\prime}\right|\right)\left[\Delta \rho(\mathbf{r})+\Delta \rho\left(\mathbf{r}^{\prime}\right)\right] \\
& +\frac{1}{2} K_{11}\left(\left|\mathbf{r}-\mathbf{r}^{\prime}\right|\right)\left[\Delta \rho^{2}(\mathbf{r})+\Delta \rho^{2}\left(\mathbf{r}^{\prime}\right)\right] \\
& +K_{12}\left(\left|\mathbf{r}-\mathbf{r}^{\prime}\right|\right) \Delta \rho(\mathbf{r}) \Delta \rho\left(\mathbf{r}^{\prime}\right)+\cdots
\end{aligned}
$$

where $\Delta \rho(\mathbf{r})=\rho(\mathbf{r})-\bar{\rho}$, and

$$
\begin{aligned}
K_{0}\left(\left|\mathbf{r}-\mathbf{r}^{\prime}\right|\right) & =K\left(\bar{\rho}, \bar{\rho} ;\left|\mathbf{r}-\mathbf{r}^{\prime}\right|\right), \\
K_{1}\left(\left|\mathbf{r}-\mathbf{r}^{\prime}\right|\right) & =\left.\frac{\partial K\left(\rho(\mathbf{r}), \rho\left(\mathbf{r}^{\prime}\right) ;\left|\mathbf{r}-\mathbf{r}^{\prime}\right|\right)}{\partial \rho(\mathbf{r})}\right|_{\bar{\rho}}, \\
K_{11}\left(\left|\mathbf{r}-\mathbf{r}^{\prime}\right|\right) & =\left.\frac{\partial^{2} K\left(\rho(\mathbf{r}), \rho\left(\mathbf{r}^{\prime}\right) ;\left|\mathbf{r}-\mathbf{r}^{\prime}\right|\right)}{\partial \rho^{2}(\mathbf{r})}\right|_{\bar{\rho}}, \\
K_{12}\left(\left|\mathbf{r}-\mathbf{r}^{\prime}\right|\right) & =\left.\frac{\partial^{2} K\left(\rho(\mathbf{r}), \rho\left(\mathbf{r}^{\prime}\right) ;\left|\mathbf{r}-\mathbf{r}^{\prime}\right|\right)}{\partial \rho(\mathbf{r}) \rho\left(\mathbf{r}^{\prime}\right)}\right|_{\bar{\rho}}, \cdots
\end{aligned}
$$

Then Eq. A1 can be evaluated as a sum of separate convolutions with kernels $K_{0}, K_{1}$, etc. WGC also demonstrated that only a few terms of the expansion in Eq.(A2) are necessary to evaluate the convolution accurately for physical systems.

The present real space method is clearly applicable to such kinetic energy functionals, provided $K_{0}(\bar{q}), K_{1}(\bar{q})$, etc., where $\bar{q}=k /\left(2 \bar{k}_{F}\right)$ and $\bar{k}_{F}=\left(3 \pi^{2} \bar{\rho}\right)^{1 / 3}$, can be fit well by 
functions of the form of Eq.(20). For the kernels other than $K_{0}$, a fitting form slightly different than Eq. (20) is necessary, because for $\bar{q} \rightarrow \infty, K_{0}(\bar{q})$ approaches a constant, as does the rational form Eq. (20), but the higher order kernels decay as $\bar{q}^{-2}$. Thus they are fit with the modified form:

$$
\frac{N_{2} \bar{q}^{2}+\cdots+N_{2(m-1)} \bar{q}^{2(m-1)}}{D_{0}+D_{2} \bar{q}^{2}+\cdots+D_{2 m} \bar{q}^{2 m}} .
$$

Functions of this form decompose into the following terms:

$$
\tilde{K}(\bar{q})=\sum_{j=1}^{m} \frac{R_{j}}{\bar{q}^{2}+S_{j}}
$$

i.e. just as in Eq. (21), but without the $q^{2}$ in the numerator. Now the $V_{j}(\mathbf{r})$ are obtained by solving a modified form of Eq. (24):

$$
\left[-\frac{1}{\left(2 \bar{k}_{F}\right)^{2}} \nabla^{2}+S_{j}\right] V_{j}(\mathbf{r})=R_{j} f(\mathbf{r}),
$$

which is still a complex Helmholtz equation. The kinetic energy functionals of WGC have three parameters, $\alpha, \beta$, and $\gamma$. Presently, only the case of $\{\alpha, \beta\}=\frac{5}{6} \pm \frac{\sqrt{5}}{6}, \gamma=2.7$, (suggested by favorable numerical tests) is considered. In Fig. ॠ, the best fit to these kernels with a rational function of order eight $(m=4)$ is shown. The quality of the fit is excellent for $K_{0}(\bar{q})$ and $K_{1}(\bar{q})$, and reasonable for the second order kernels $K_{11}(\bar{q}), K_{12}(\bar{q})$. WGC have shown that second order terms contribute much smaller parts of $T_{K}[\rho]$ (which is already a small part of the total energy) than the zeroth and first order terms, and thus a higher fraction of error can be tolerated in these terms. The $P_{j}, Q_{j}$ of the decompositions of the fits to $K_{0}$ (Eq.(21)) and the $R_{j}, S_{j}$ of the fits to $K_{1}, K_{11}$, and $K_{12}$ (Eq.(A4)) are given in Table III.

[1] P. Hohenberg and W. Kohn, Phys. Rev. 136, B864 (1964).

[2] W. Kohn and L. J. Sham, Phys. Rev. 140, A1133 (1965).

[3] Y. A. Wang and E. A. Carter, in Theoretical Methods in Condensed Phase Chemistry, edited by S. D. Schwartz, Progress in Theoretical Chemistry and Physics, (Kluwer, 2000) p. 117.

[4] L.-W. Wang and M. P. Teter, Phys. Rev. B 45, 13196 (1992).

[5] F. Perrot, J. Phys.: Cond. Mat. 6, 431 (1994).

[6] E. Chacón, J. E. Alvarellos, and P. Tarazona, Phys. Rev. B 32, 7868 (1985). 
[7] E. Smargiassi and P. A. Madden, Phys. Rev. B 49, 5220 (1994).

[8] Y. A. Wang, N. Govind, and E. A. Carter, Phys. Rev. B 58, 13465 (1998).

[9] Y. A. Wang, N. Govind, and E. A. Carter, Phys. Rev. B 60, 16350 (1999).

[10] L. H. Thomas, Proc. Cambridge Philos. Soc. 23, 542 (1926); E. Fermi, Z. Phys. 48, 73 (1928).

[11] C. F. von Weizsäcker, Z. Phys. 96, 431 (1935).

[12] J. Lindhard, Kgl. Danske Videnskab. Selskab., Mat. Fys. Medd. 28, 8 (1954).

[13] C. Herring, Phys. Rev. A 34, 2614 (1986).

[14] Y. Saad, Iterative methods for sparse linear systems (Boston : PWS Pub. Co., 1996.)

[15] W. Harrison, Solid State Theory (Dover, New York, N.Y., 1980.)

[16] R. W. Freund, SIAM J. Sci. Stat. Comp., 13(1), 425 (1994).

[17] R. W. Freund and N. Nachtigal, Numer. Math. 60, 315 (1991); H. van der Vorst and J. Melissen, IEEE Trans. Mag., 26(2), 706 (1990).

[18] L. Goodwin, R. J. Needs, and V. Heine, J. Phys. Cond. Matter 2, 351 (1990).

[19] J. P. Perdew and A. Zunger, Phys. Rev. B 23, 5048 (1981).

\section{FIGURES}




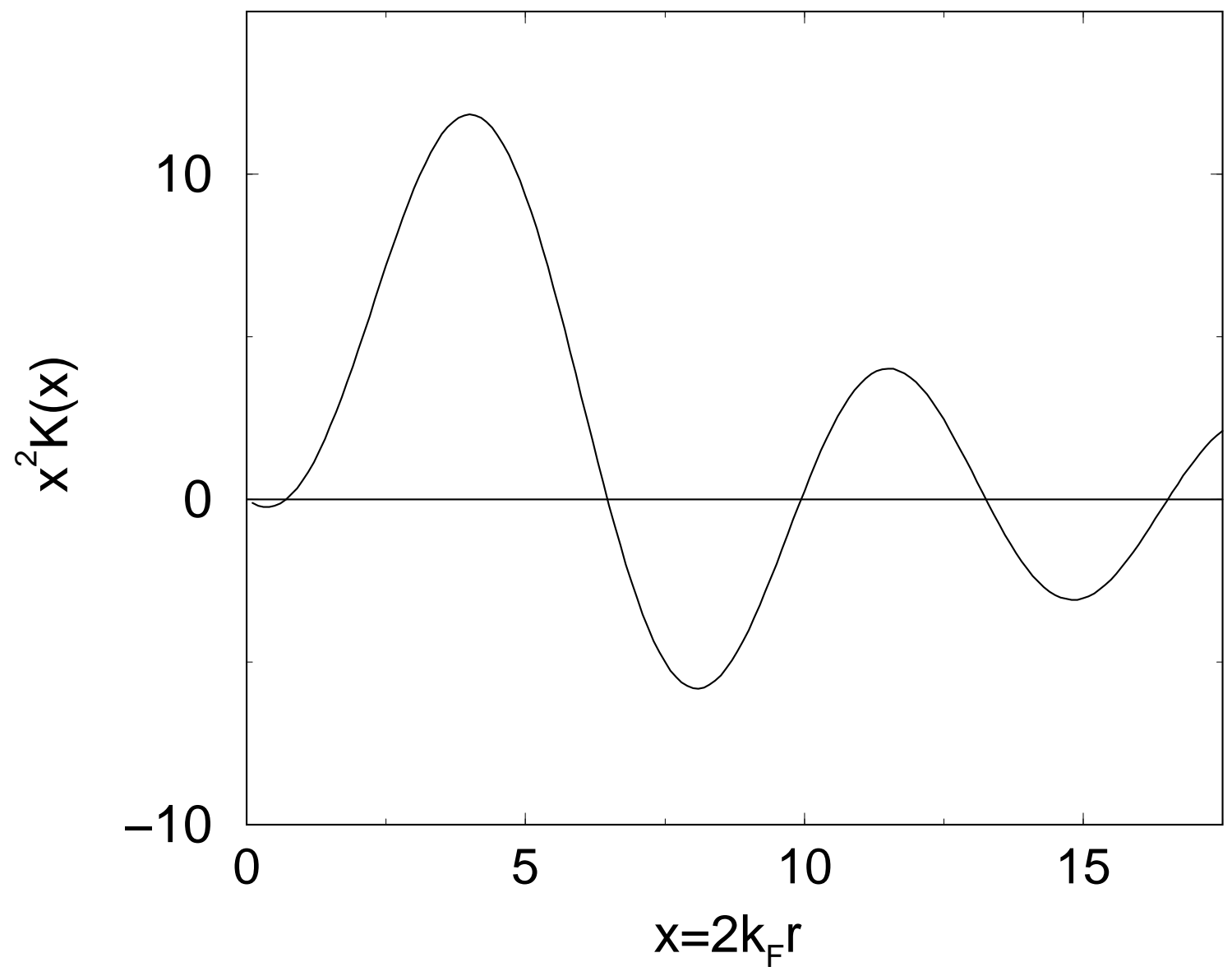

FIG. 1: Kinetic energy functional kernel $K(x)$, where $x=2 k_{F} r$, multiplied by $x^{2}$, showing its long-ranged nature. 


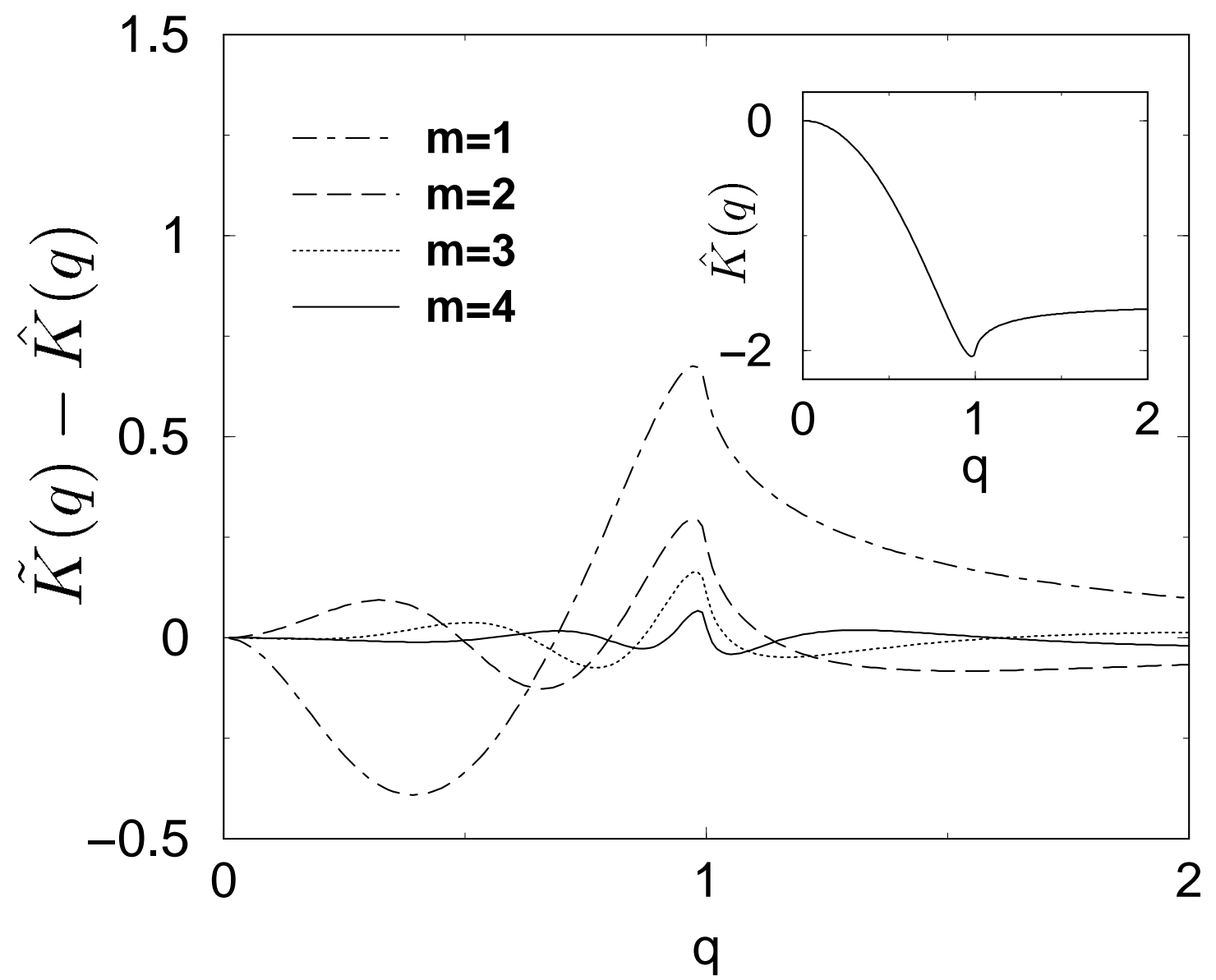

FIG. 2: The difference between the rational fitting functions $\tilde{K}(q)$ (Eq. 20) ) and $\hat{K}(q)$ (Eq.(99) with the number of terms ranging from $m=1$ to $m=4$. The inset shows $\hat{K}(q)$. 


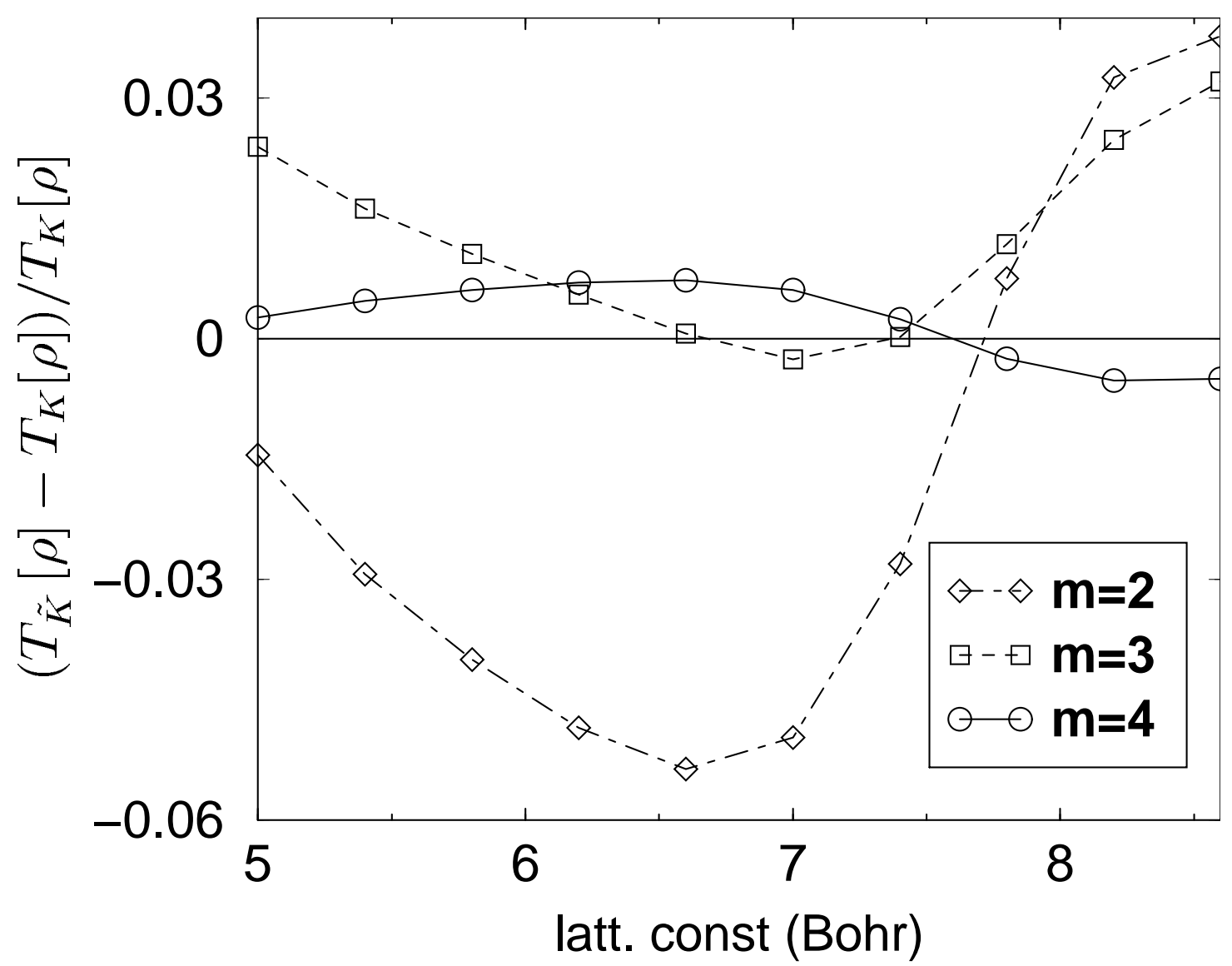

FIG. 3: The fractional deviation of $T_{\tilde{K}}[\rho]$ from $T_{K}[\rho]$ for densities $\rho(\mathbf{r})$ obtained from bulk Al calculations (see text). 


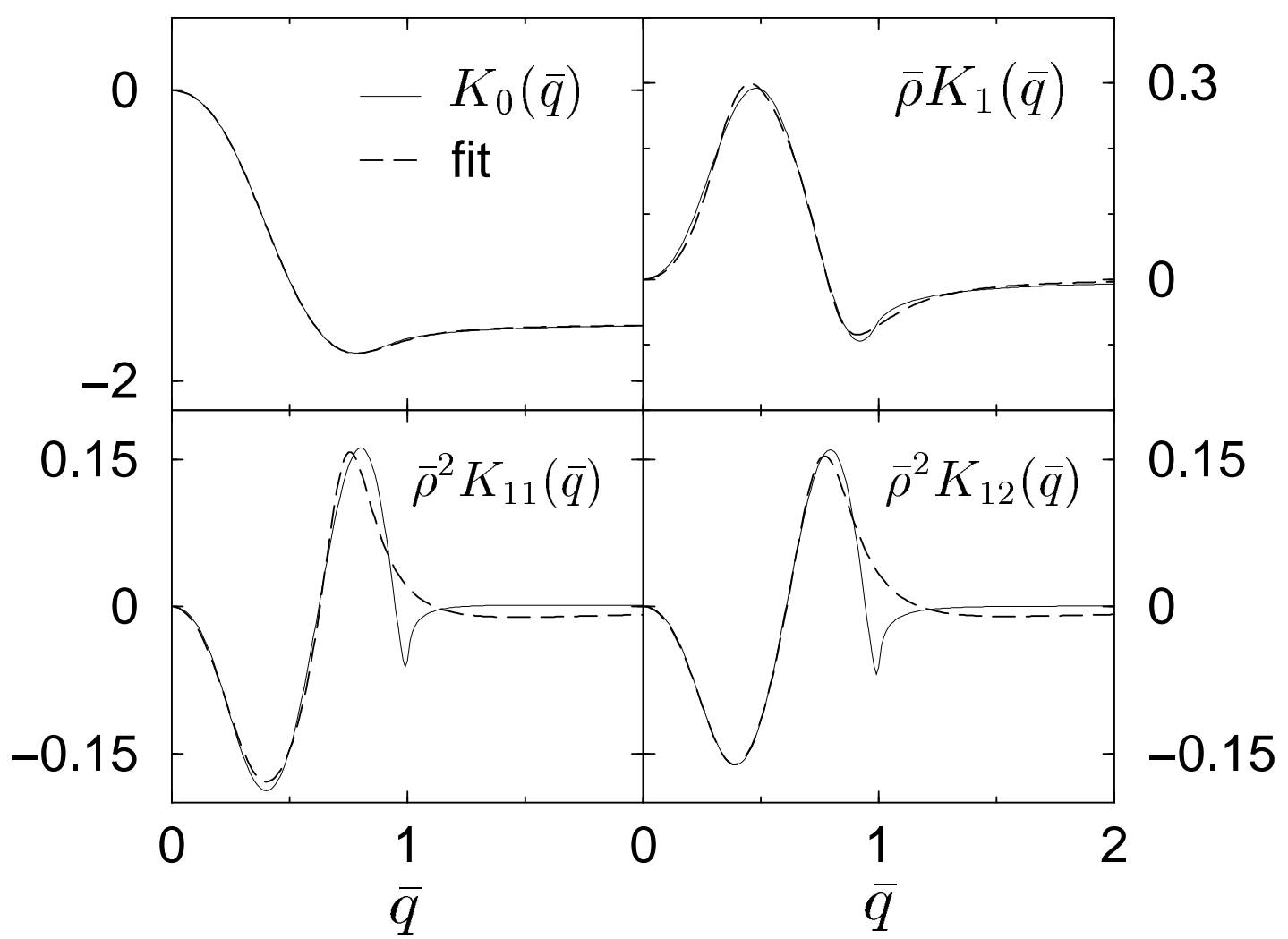

FIG. 4: The fitting of the various density-dependent kernels of WGC[9] by the forms of Eqs. (20) and (A4) with $m=4$. Notice the vastly increased scale for $K_{1}, K_{11}$, and $K_{12}$, necessary to show their features, since they are negligible on the scale of $K_{0}$.

\section{TABLES}

TABLE I: Optimized fitting parameters for $\tilde{K}(q)$ with an order-eight $(m=4)$ rational function of Eq.(21). The parameters with even indices $j=2,4$ are complex conjugates of the ones given:

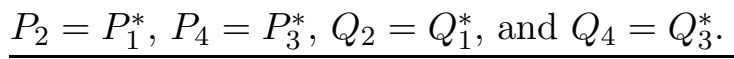

\begin{tabular}{c|cc}
\hline \hline & $j=1$ & $j=3$ \\
\hline$P_{j}$ & $0.026696+i 0.145493$ & $-0.826696+i 0.691930$ \\
$Q_{j}$ & $-0.818245-i 0.370856$ & $0.343051-i 0.689646$ \\
\hline \hline
\end{tabular}


TABLE II: Lattice constant $a_{0}$, in $\AA$, and bulk modulus B, in GPa, for bulk fcc aluminum with the WGC kinetic energy functional 9] with a density-independent kernel, and with $\{\alpha, \beta\}=\frac{5}{6} \pm \frac{\sqrt{5}}{6}$, compared to those values determined with the present real-space method with $m=2,3$, and 4 . Also shown is $a_{0}$ and B from the Kohn-Sham (KS) calculation.

\begin{tabular}{rccccc}
\hline \hline & $\mathrm{KS}$ & reciprocal space & $m=4$ & $m=3$ & $m=2$ \\
\hline$a_{0}(\AA)$ & 4.027 & 4.035 & 4.030 & 4.045 & 4.063 \\
$\mathrm{~B}(\mathrm{GPa})$ & 68.5 & 71.9 & 72.3 & 68.6 & 68.0 \\
\hline \hline
\end{tabular}

TABLE III: Optimized fitting parameters $P_{j}, Q_{j}, R_{j}$, and $S_{j}$ of Eqs. (21) and (A5) for fits to the kernels $K_{0}(\bar{q}), K_{1}(\bar{q}), K_{11}(\bar{q})$, and $K_{12}(\bar{q})$ of the WGC density-dependent kinetic energy functional with $\{\alpha, \beta\}=\frac{5}{6} \pm \frac{\sqrt{5}}{6}$ and $\gamma=2.7$. The parameters with even indices, $j=2,4$, are complex conjugates of the ones given: $X_{2}=X_{1}^{*}$, and $X_{4}=X_{3}^{*}$, where $X=P, Q, R$, or $S$.

\begin{tabular}{cr|cc}
\hline \hline & & $j=1$ & $j=3$ \\
\hline & $P_{j}$ & $0.108403+i 0.079657$ & $-0.908403+i 0.439708$ \\
$K_{0}$ & $Q_{j}$ & $-0.470923-i 0.465392$ & $0.066051-i 0.259678$ \\
& $R_{j}$ & $-0.030515+i 0.015027$ & $0.028915-i 0.008817$ \\
$\bar{\rho} K_{1}$ & $S_{j}$ & $-0.597793-i 0.294130$ & $-0.087917-i 0.164937$ \\
& $R_{j}$ & $0.008907-i 0.032841$ & $-0.034974+i 0.009116$ \\
$\bar{\rho}^{2} K_{11}$ & $S_{j}$ & $-0.537986-i 0.233840$ & $-0.041565-i 0.196662$ \\
& $R_{j}$ & $0.012423-i 0.034421$ & $-0.031907+i 0.007392$ \\
$\bar{\rho}^{2} K_{12}$ & $S_{j}$ & $-0.511699-i 0.266195$ & $-0.034031-i 0.188927$ \\
\hline \hline
\end{tabular}

